\title{
Robustness of Forecasting for Autoregressive Time Series with Bilinear Distortions
}

\author{
Yuriy Kharin and Olga Radzieuskaya \\ Belarusian State University, Minsk
}

\begin{abstract}
The paper is devoted to the investigation of bilinear stochastic time series model $\mathrm{BL}(\mathrm{p}, 0,1,1)$. The linear autoregressive forecasting statistic is considered under the mean-square risk criterion; its robustness under bilinear distortions is evaluated.
\end{abstract}

Keywords: Autoregression, Bilinear Time Series, Mean-Square Risk, Robustness.

\section{Introduction}

There is a growing interest in the investigation of nonlinear time series models that is caused by nonlinearity of real processes. This paper contributes to the development of the particular nonlinear time series model analysis, the bilinear time series model.

Bilinear time series model was proposed by Granger and Andersen in 1978 (Fan and Yao, 2003; Granger and Andersen, 1978) as an alternative to the linear time series model. The model gives a possibility to describe "sufficiently large stochastic outbursts" that often appear in applications to seismological and financial data analysis. Since the time of introduction, quite a lot of researches has been carried out in the investigation of the bilinear model; stationarity, casuality, invertibility conditions have been found, moreover, parameter estimation techniques, tests for bilinearity and other solutions have been presented and studied (Granger and Andersen, 1978; Rao and Gabr, 1984; Terdik, 1999).

This paper points at the problem of the bilinear time series $\mathrm{BL}(\mathrm{p}, 0,1,1)$ prediction. The forecasting statistic of the well-known linear autoregressive model AR(p) is applied; its robustness under bilinear distortions is studied.

The paper is organized according to the following structure. In Section 2 the bilinear model BL(p,0,1,1) and the corresponding linear model AR(p) are defined and some their properties are listed. Further, the linear forecasting for bilinear time series is considered in Section 3, and its robustness is studied in Section 4. Section 5 presents the results of numerical experiments.

\section{Models BL(p, 0, 1, 1), AR(p) and their Properties}

The bilinear model BL(p, $0,1,1)$ is a simple nonlinear modification of the well-known and intensively used linear autoregressive model $\mathrm{AR}(\mathrm{p})$ of the order $p$. Therefore, it is quite reasonable to consider these models together and apply some earlier obtained results of linear model $\mathrm{AR}(\mathrm{p})$ to nonlinear model $\mathrm{BL}(\mathrm{p}, 0,1,1)$. 
The time series $\left\{x_{t}^{0}\right\}_{t \in Z}$ is said to correspond with autoregressive model $\operatorname{AR}(\mathrm{p})$ if it satisfies the linear stochastic difference equation

$$
x_{t}^{0}=\sum_{j=1}^{p} \alpha_{j} x_{t-j}^{0}+u_{t}, \quad t \in Z,
$$

where $Z=\{\ldots,-1,0,+1, \ldots\}$ is the set of integers, $\left\{u_{t}\right\}_{t \in Z}$ are independent identically distributed by $N\left(0, \sigma^{2}\right),\left\{\alpha_{j}\right\}_{j=1}^{p}$ are autoregressive coefficients.

Respectively, the time series $\left\{x_{t}\right\}_{t \in Z}$ is said to correspond with bilinear model BL(p, $0,1,1)$ if it satisfies the bilinear stochastic difference equation

$$
x_{t}=\sum_{j=1}^{p} \alpha_{j} x_{t-j}+\beta x_{t-1} u_{t-1}+u_{t}, \quad t \in Z,
$$

where $\beta$ is the bilinearity coefficient. Note, if $\beta=0$, then the models (1) and (2) are the same.

Further only stationary models $\mathrm{AR}(\mathrm{p})(1)$ and $\mathrm{BL}(\mathrm{p}, 0,1,1)(2)$ will be considered. The conditions (Anderson, 1994; Rao and Gabr, 1984)

$$
\rho(A)<1 \quad \text { and } \quad \rho\left(A \otimes A+\sigma^{2} B \otimes B\right)<1
$$

provide their stationarity respectively. Here the matrices $A$ and $B$ are

$$
A=\left(\begin{array}{ccccc}
-\alpha_{1} & -\alpha_{2} & \ldots & -\alpha_{p-1} & -\alpha_{p} \\
1 & 0 & \ldots & 0 & 0 \\
0 & 1 & \ldots & 0 & 0 \\
\ldots & \ldots & \ldots & \ldots & \ldots \\
0 & 0 & \ldots & 1 & 0
\end{array}\right) \in \mathbb{R}^{p}, \quad B=\left(\begin{array}{ccccc}
1 & 0 & \ldots & 0 & 0 \\
0 & 0 & \ldots & 0 & 0 \\
0 & 0 & \ldots & 0 & 0 \\
\ldots & \ldots & \ldots & \ldots & \ldots \\
0 & 0 & \ldots & 0 & 0
\end{array}\right) \in \mathbb{R}^{p}
$$

and $\otimes$ means the Kronecker product.

Note that the model (1) defines zero-mean time series, while the model (2) has the mean equal to

$$
\mathrm{E}\left\{x_{t}\right\}=\frac{\beta \sigma^{2}}{1-\sum_{j=1}^{p} \alpha_{j}} .
$$

Let's define the second order moments of $\mathrm{AR}(\mathrm{p})$ and $\mathrm{BL}(\mathrm{p}, 0,1,1)$ time series respectively:

$$
c_{0}(s)::=\mathrm{E}\left\{x_{t}^{0} x_{t-s}^{0}\right\}, \quad c(s)::=\mathrm{E}\left\{x_{t} x_{t-s}\right\}, \quad s \in Z .
$$

It is known (Anderson, 1994) that the moments of the $\operatorname{AR}(\mathrm{p})$ process $c_{0}(s), s \in Z$, satisfy the Yule-Walker system of equations. The second order moments of bilinear time series can be found as a solution of the Yule-Walker-like linear system of equations as well.

Introduce the matrix notation:

$$
\begin{gathered}
W=W_{1}+I_{p+1}+W_{2} \in R^{(p+1) \times(p+1)}, \\
W_{1}=\left(\begin{array}{ccccc}
0 & -\alpha_{1} & -\alpha_{2} & \ldots & -\alpha_{p} \\
0 & -\alpha_{2} & -\alpha_{3} & \ldots & 0 \\
\ldots & \ldots & \ldots & \ldots & \ldots \\
0 & -\alpha_{p} & 0 & \ldots & 0 \\
0 & 0 & 0 & \ldots & 0
\end{array}\right), \quad W_{2}=\left(\begin{array}{ccccc}
0 & 0 & \ldots & 0 & 0 \\
-\alpha_{1} & 0 & \ldots & 0 & 0 \\
\ldots & \ldots & \ldots & \ldots & \ldots \\
-\alpha_{p-1}-\alpha_{p-2} & \ldots & 0 & 0 \\
-\alpha_{p} & -\alpha_{p-1} & \ldots & -\alpha_{1} & 0
\end{array}\right),
\end{gathered}
$$


$i_{1}=(1,0, \ldots, 0) \in \mathbb{R}^{p+1}$ is the first column of the identity matrix $I_{p+1} \in \mathbb{R}^{(p+1) \times(p+1)}$,

$$
\begin{gathered}
\mathbb{1}_{p+1}=(1)_{i, j=1}^{p+1} \in \mathbb{R}^{(p+1) \times(p+1)}, \quad c=(c(0), c(1), \ldots, c(p))^{\prime} \in \mathbb{R}^{p+1}, \\
f=\left(1+\frac{1+\alpha_{1}}{1-\sum_{j=1}^{p} \alpha_{j}}, \frac{2}{1-\sum_{j=1}^{p} \alpha_{j}}, \frac{1}{1-\sum_{j=1}^{p} \alpha_{j}}, \ldots, \frac{1}{1-\sum_{j=1}^{p} \alpha_{j}}\right)^{\prime} \in \mathbb{R}^{p+1} .
\end{gathered}
$$

Lemma 1. The second order moments of the stationary bilinear time series $B L(p, 0,1,1)$ satisfy the following equations:

$$
\begin{gathered}
\left(1-\beta^{2} \sigma^{2}\right) c(0)=\sum_{j=1}^{p} \alpha_{j} c(j)+\sigma^{2}+\beta^{2} \sigma^{4}+\frac{\beta^{2} \sigma^{4}\left(1+\alpha_{1}\right)}{1-\sum_{j=1}^{p} \alpha_{j}}, \\
c(1)=\sum_{j=1}^{p} \alpha_{j} c(j-1)+\frac{2 \beta^{2} \sigma^{4}}{1-\sum_{j=1}^{p} \alpha_{j}}, \quad c(s)=\sum_{j=1}^{p} \alpha_{j} c(j-s)+\frac{\beta^{2} \sigma^{4}}{1-\sum_{j=1}^{p} \alpha_{j}}, \quad s=2, \ldots, p,
\end{gathered}
$$

or in the matrix form:

$$
\left(W-\beta^{2} \sigma^{2} i_{1} i_{1}^{\prime}\right) \cdot c=\sigma^{2} i_{1}+\beta^{2} \sigma^{4} f
$$

if $|W| \neq 0$ and $\beta^{2} \sigma^{2} W^{-1}(1,1) \neq 1$, then

$$
c=W^{-1}\left(I_{p+1}-\beta^{2} \sigma^{2} i_{1} i_{1}^{\prime} W^{-1}\right)^{-1}\left(\sigma^{2} i_{1}+\beta^{2} \sigma^{4} f\right) .
$$

Proof. Calculate mathematical expectation of both sides of the difference equation (2) multiplied by $x_{t-s}$ :

$$
\mathrm{E}\left\{x_{t} x_{t-s}\right\}=\sum_{j=1}^{p} \alpha_{j} \mathrm{E}\left\{x_{t-j} x_{t-s}\right\}+\beta \mathrm{E}\left\{x_{t-1} x_{t-s} u_{t-1}\right\}+\mathrm{E}\left\{x_{t-s} u_{t}\right\}, \quad s=0, \ldots, p .
$$

In particular, when $s=0$,

$$
\mathrm{E}\left\{x_{t}^{2}\right\}=\sum_{j=1}^{p} \alpha_{j} \mathrm{E}\left\{x_{t-j} x_{t}\right\}+\beta \mathrm{E}\left\{x_{t-1} x_{t} u_{t-1}\right\}+\mathrm{E}\left\{x_{t} u_{t}\right\}
$$

Because of the independence property for $\left\{u_{t}\right\}_{t \in Z}, \mathrm{E}\left\{x_{t-s} u_{t}\right\}=0, s=1, \ldots, p$. Moreover, $\mathrm{E}\left\{x_{t} u_{t}\right\}=\sigma^{2}$.

It is easily obtained that

$$
\mathrm{E}\left\{x_{t-k} x_{t} u_{t}\right\}=\frac{\beta \sigma^{4}}{1-\sum_{j=1}^{p} \alpha_{j}}, \quad k=1, \ldots, p, \quad \mathrm{E}\left\{x_{t}^{2} u_{t}\right\}=\frac{2 \beta \sigma^{4}}{1-\sum_{j=1}^{p} \alpha_{j}} .
$$

The proof of the last statement on $\mathrm{E}\left\{x_{t}^{2} u_{t}\right\}$ is based on the equation

$$
\mathrm{E}\left\{x_{t}^{2} u_{t}^{2}\right\}=\sigma^{2} \mathrm{E}\left\{x_{t}^{2}\right\}+2 \sigma^{4} .
$$

By substituting the values $\mathrm{E}\left\{x_{t-k} x_{t} u_{t}\right\}, k=0, \ldots, p$, into (3), we obtain the statement of the lemma at first in scalar form and then represent it in matrix form.

Together with exact values of the second order moments, the asymptotic expansion at $\beta \rightarrow 0$ is useful. 
Corollary 1. Under the assumptions of Lemma 1 , if $|W| \neq 0$ and $\beta \rightarrow 0$, then

$$
c=\sigma^{2} W^{-1} i_{1}+\beta^{2} \sigma^{4}\left(W^{-1} f+W^{-1}(1,1) W^{-1} i_{1}\right)+o\left(\beta^{2}\right) \mathbb{1}_{p+1} ;
$$

in particular,

$$
c(0)=\sigma^{2} W^{-1}(1,1)+\beta^{2} \sigma^{4}\left(i_{1}^{\prime} W^{-1} f+\left(W^{-1}(1,1)\right)^{2}\right)+o\left(\beta^{2}\right) .
$$

For further analysis, it is more convenient to use the matrix form of the models $\operatorname{AR}(\mathrm{p})$ and $\mathrm{BL}(\mathrm{p}, 0,1,1)$. The following lemma gives this representation.

Introduce the notation:

$$
\begin{gathered}
S_{1}=\left(\begin{array}{cccc}
1 & -\alpha_{1} \ldots & -\alpha_{p-1} \\
0 & 1 & \ldots & -\alpha_{p-2} \\
\ldots & \ldots & \ldots & \ldots \\
0 & 0 & \ldots & 1
\end{array}\right) \in \mathbb{R}^{p \times p}, \quad S_{2}=\left(\begin{array}{cccc}
\alpha_{p} & 0 & \ldots & 0 \\
\alpha_{p-1} & \alpha_{p} & \ldots & 0 \\
\ldots & \ldots & \ldots & \ldots \\
\alpha_{1} & \alpha_{2} & \ldots & \alpha_{p}
\end{array}\right) \in \mathbb{R}^{p \times p}, \\
X_{n}^{m}=\left(x_{m}, \ldots, x_{n}\right)^{\prime} \in \mathbb{R}^{m-n+1}, \quad X_{n}^{0^{m}}=\left(x_{m}^{0}, \ldots, x_{n}^{0}\right)^{\prime} \in \mathbb{R}^{m-n+1}, \\
U_{n}^{m}=\left(u_{m}, \ldots, u_{n}\right)^{\prime} \in \mathbb{R}^{m-n+1}, \quad<X>_{n}^{m}=\left(x_{m} u_{m}, \ldots, x_{n} u_{n}\right)^{\prime} \in \mathbb{R}^{m-n+1} .
\end{gathered}
$$

Lemma 2. The stochastic difference equations (1) and (2) can be represented in the matrix form:

$$
\begin{aligned}
S_{1} X_{T+1}^{0^{T+p}} & =S_{2} X_{T-p+1}^{0^{T}}+U_{T+1}^{T+p}, \\
S_{1} X_{T+1}^{T+p} & =S_{2} X_{T-p+1}^{T}+\beta<X U>_{T}^{T+p-1}+U_{T+1}^{T+p} .
\end{aligned}
$$

Proof. The equations (4) and (5) follow from the difference equations (1), (2) and the notations that has been introduced above.

\section{Autoregressive Forecasting of Bilinear Time Series}

The significant and complicated problem is to find a forecasting statistic $\hat{x}_{T+\tau}$ as an estimator of the future value $x_{T+\tau}$ of the time series optimal w.r.t. some criterion by the observed history $X=\left\{x_{1}, \ldots, x_{T}\right\}$ of length $T$. Here $\tau, \tau=1, \ldots, p$, is a horizon of prediction. As an optimality criterion we will use a set of scalar risks

$$
r(\tau)=\mathrm{E}\left\{\left(\hat{x}_{T+\tau}-x_{T+\tau}\right)^{2}\right\} \geq 0
$$

and the $(p \times p)$-matrix risk

$$
R=\left(\mathrm{E}\left\{\left(\hat{x}_{T+\tau_{1}}-x_{T+\tau_{1}}\right)\left(\hat{x}_{T+\tau_{2}}-x_{T+\tau_{2}}\right)\right\}\right)_{\tau_{1}, \tau_{2}=1}^{p} \in \mathbb{R}^{p \times p} .
$$

For the time series corresponded to the model AR(p) (1) the problem of forecasting w.r.t. the mean-square risk optimality criterion is solved. The forecasted values, which provide minimum of defined earlier risks, can be found from the following system of equations (Anderson, 1994):

$$
S_{1} \hat{X}_{T+1}^{0 T+p}=S_{2} X_{T-p+1}^{0^{T}},
$$

where $\hat{X}_{T+1}^{0^{T+p}}=\left(\hat{x}_{T+1}, \ldots, \hat{x}_{T+p}\right)^{\prime}$ is the $p$-vector of forecasted values, $X_{T-p+1}^{0^{T}}=$ $\left(x_{T-p+1}, \ldots, x_{T}\right)^{\prime}$ is the $p$-vector of the last $p$ observed values, matrices $S_{1}, S_{2} \in \mathbb{R}^{p \times p}$ have been defined earlier. 
Theorem 1. The forecasting statistic defined by (6) for the stationary model AR(p) (1) or (4) is unbiased; moreover, its matrix and scalar risks are defined by the following formulas (Anderson, 1994):

$$
\begin{gathered}
R=\sigma^{2}\left(S_{1}^{\prime} S_{1}\right)^{-1} \\
r(\tau)=\sigma^{2} \sum_{i=1}^{\tau}\left(S_{1}^{-1}(1, i)\right)^{2}, \quad \tau=1, \ldots, p .
\end{gathered}
$$

Let us assume a low level of bilinear distortions in the model $\mathrm{BL}(\mathrm{p}, 0,1,1)$ that means the coefficient $\beta$ to be sufficiently small. So it is reasonable to use the autoregressive forecasting statistic (6) to predict future values of bilinear time series. Let us define the forecasting statistic from the equation equivalent to (6):

$$
S_{1} \hat{X}_{T+1}^{T+p}=S_{2} X_{T-p+1}^{T} .
$$

Theorem 2. The forecasting statistic (7) for the stationary model $B L(p, 0,1,1)(2)$ or (5) is biased:

$$
\mathrm{E}\left\{X_{T+1}^{T+p}-\hat{X}_{T+1}^{T+p}\right\}=\beta \sigma^{2} S_{1}^{-1}(1,1, \ldots, 1)^{\prime} \neq(0,0, \ldots, 0)^{\prime} \in \mathbb{R}^{p} .
$$

Proof. From (5) and (7)

$$
X_{T+1}^{T+p}-\hat{X}_{T+1}^{T+p}=\beta S_{1}^{-1}<X U>_{T}^{T+p-1}+S_{1}^{-1} U_{T+1}^{T+p} .
$$

Therefore, after calculation of mathematical expectation we obtain that the forecasting statistic (7) is biased.

However, it needs to be noted that for the low level of bilinear distortions $\beta$ the forecasting statistic (7) tends to be unbiased.

Theorem 3. For the stationary model $B L(p, 0,1,1)$ defined by (2) or (5) the autoregressive forecasting statistic (7) has the following matrix mean-square risk:

$$
\begin{aligned}
R= & \sigma^{2}\left(S_{1}\right)^{-1}\left(S_{1}^{-1}\right)^{\prime}+\beta^{2} \sigma^{2} c(0)\left(S_{1}\right)^{-1}\left(S_{1}^{-1}\right)^{\prime}+\beta^{2} \sigma^{4}\left(S_{1}\right)^{-1}\left(\mathbb{1}_{p \times p}+I_{p}\right)\left(S_{1}^{-1}\right)^{\prime} \\
& +\frac{2 \beta^{2} \sigma^{4}}{1-\sum_{j=1}^{p} \alpha_{j}}\left(S_{1}\right)^{-1}\left(I_{p}^{-1,0,1}-I_{p}\right)\left(S_{1}^{-1}\right)^{\prime},
\end{aligned}
$$

where $I_{p}^{-1,0,1} \in \mathbb{R}^{p \times p}$ is the matrix with identities only under and above diagonal, while other elements are zeros.

Proof. From (5) and (7)

$$
S_{1}\left(X_{T+1}^{T+p}-\hat{X}_{T+1}^{T+p}\right)=\beta<X U>_{T}^{T+p-1}+U_{T+1}^{T+p} .
$$

Therefore,

$$
\begin{gathered}
S_{1} \mathrm{E}\left\{\left(\hat{X}_{T+1}^{T+p}-X_{T+1}^{T+p}\right)\left(\hat{X}_{T+1}^{T+p}-X_{T+1}^{T+p}\right)^{\prime}\right\} S_{1}^{\prime}=S_{1} R S_{1}^{\prime} \\
=\beta^{2} \mathrm{E}\left\{<X U>_{T}^{T+p-1}\left(<X U>_{T}^{T+p-1}\right)^{\prime}\right\}+\beta \mathrm{E}\left\{<X U>_{T}^{T+p-1}\left(U_{T+1}^{T+p}\right)^{\prime}\right\} \\
+\beta \mathrm{E}\left\{U_{T+1}^{T+p}\left(<X U>_{T}^{T+p-1}\right)^{\prime}\right\}+\mathrm{E}\left\{U_{T+1}^{T+p}\left(U_{T+1}^{T+p}\right)^{\prime}\right\} .
\end{gathered}
$$

By calculating the mathematical expectation of every element in the right part of this expression and relying on Lemma 1 , due to $\left|S_{1}\right| \neq 0$, we get the required statement of the theorem. 
Corollary 2. Under the conditions of Theorem 3, the scalar risks of the forecasting statistic (7) are

$$
\begin{aligned}
& r(\tau)=\sigma^{2} \sum_{j=1}^{\tau}\left(S_{1}^{-1}(1, j)\right)^{2}+\beta^{2} \sigma^{2} c(0) \sum_{j=1}^{\tau}\left(S_{1}^{-1}(1, j)\right)^{2}+\beta^{2} \sigma^{4}\left(\sum_{j=1}^{\tau}\left(S_{1}^{-1}(1, j)\right)^{2}+\right. \\
& \left.+\left(\sum_{j=1}^{\tau} S_{1}^{-1}(1, j)\right)^{2}+\frac{4}{1-\sum_{j=1}^{p} \alpha_{j}} \sum_{j=1}^{\tau-1} S_{1}^{-1}(1, j) S_{1}^{-1}(1, j+1)\right), \quad \tau=1, \ldots, p .
\end{aligned}
$$

Proof. According to the definition, the scalar risks are the diagonal elements of the matrix risk: $r(\tau)=R(p-\tau+1, p-\tau+1), \tau=1, \ldots, p$. Because of the triangularity of $S_{1}^{-1}$ as well as $S_{1}$, we obtain the formula (9)

Corollary 3. Under the conditions of Theorem 1 , if $|W| \neq 0$, then the matrix and the scalar mean-square risks satisfy the following asymptotic expansions at $\beta \rightarrow 0$ :

$$
\begin{gathered}
R=\sigma^{2} S_{1}^{-1}\left(S_{1}^{-1}\right)^{\prime}+\beta^{2} \sigma^{4}\left(W^{-1}(1,1) S_{1}^{-1}\left(S_{1}^{-1}\right)^{\prime}+S_{1}^{-1}\left(\mathbb{1}_{p \times p}+I_{p}\right)\left(S_{1}^{-1}\right)^{\prime}\right. \\
\left.+\frac{2}{1-\sum_{j=1}^{p} \alpha_{j}} S_{1}^{-1}\left(I_{p}^{-1,0,1}-I_{p}\right)\left(S_{1}^{-1}\right)^{\prime}\right)+o\left(\beta^{2}\right) \mathbb{1}_{p \times p} \\
r(\tau)=\sigma^{2} \sum_{j=1}^{\tau}\left(S_{1}^{-1}(1, j)\right)^{2}+\beta^{2} \sigma^{4}\left(\left(W^{-1}(1,1)+1\right) \sum_{j=1}^{\tau}\left(S_{1}^{-1}(1, j)\right)^{2}+\left(\sum_{j=1}^{\tau} S_{1}^{-1}(1, j)\right)^{2}\right. \\
\left.+\frac{4}{1-\sum_{j=1}^{p} \alpha_{j}} \sum_{j=1}^{\tau-1} S_{1}^{-1}(1, j) S_{1}^{-1}(1, j+1)\right)+o\left(\beta^{2}\right), \quad \tau=1, \ldots, p
\end{gathered}
$$

Proof. The proof is based on the expansion of the matrix (8) and the scalar (11) risks with neglected remainders of the expansions

\section{Robustness of the Autoregressive Forecasting Statistic under Bilinear Distortions}

The autoregressive forecasting statistic (7) applied to bilinear time series prediction ignores special structural features caused by the bilinearity. Therefore, we must be cautious while using this statistic and believe in the low level of bilinear distortions. Consider the problem of robustness and adequate use of the autoregressive forecasting statistic.

Let us define some functionals of the forecast robustness. Assume that the bilinearity level $\beta \in\left[-\beta_{+}, \beta_{+}\right]$, where $\beta_{+}>0$ is a maximum absolute level of distortions.

The instability coefficient (Kharin, 1996) is said to be the relative increment of the guaranteed upper risk $r_{+}(\tau)$ to the risk of the nondistorted times series $r_{0}(\tau)=\left.r(\tau)\right|_{\beta=0}$ :

$$
\kappa(\tau)=\left(r_{+}(\tau)-r_{0}(\tau)\right) / r_{0}(\tau), \quad r_{+}(\tau)=\sup _{\beta \in\left[-\beta_{+}, \beta_{+}\right]} r(\tau) .
$$


The $\delta$-critical distortion level (Kharin, 1996) is said to be the maximal admissible bilinearity level with the instability coefficient not greater than the given $\delta>0$ :

$$
\beta^{+}(\delta, \tau)=\sup _{\kappa(\tau) \leq \delta} \beta_{+} .
$$

The less instability coefficient $\kappa(\tau)$ and the greater $\delta$-critical distortion level $\beta^{+}(\delta, \tau)$ the autoregressive statistic is more robust under the bilinear distortions.

Theorem 4. For the stationary bilinear model $B L(p, 0,1,1)(2)$, if $|W| \neq 0$, then the instability coefficient and the $\delta$-critical distortion level have the following asymptotic representations at $\beta \rightarrow 0$ :

$$
\begin{aligned}
\kappa(\tau)= & \frac{\beta_{+}^{2} \sigma^{2}}{\sum_{j=1}^{\tau}\left(S_{1}^{-1}(1, j)\right)^{2}}\left(\left(W^{-1}(1,1)+1\right) \sum_{j=1}^{\tau}\left(S_{1}^{-1}(1, j)\right)^{2}+\left(\sum_{j=1}^{\tau} S_{1}^{-1}(1, j)\right)^{2}\right. \\
& \left.+\frac{4}{1-\sum_{j=1}^{p} \alpha_{j}} \sum_{j=1}^{\tau-1} S_{1}^{-1}(1, j) S_{1}^{-1}(1, j+1)\right)+o\left(\beta_{+}^{2}\right) \\
\beta^{+}(\delta, \tau) \approx & \frac{\delta^{1 / 2} \sigma^{-1}}{\left(\sum_{j=1}^{\tau}\left(S_{1}^{-1}(1, j)\right)^{2}\right)^{-1 / 2}}\left(\left(W^{-1}(1,1)+1\right) \sum_{j=1}^{\tau}\left(S_{1}^{-1}(1, j)\right)^{2}+\left(\sum_{j=1}^{\tau} S_{1}^{-1}(1, j)\right)^{2}\right. \\
& \left.+\frac{4}{1-\sum_{j=1}^{p} \alpha_{j}} \sum_{j=1}^{\tau-1} S_{1}^{-1}(1, j) S_{1}^{-1}(1, j+1)\right)^{-1 / 2}
\end{aligned}
$$

Proof. The theorem is proved by substitution asymptotic expansions of the scalar risks obtained in Corollary 3 into the definitions of the instability coefficient and the $\delta$-critical distortion level.

Thus, for the given $\delta>0$ we can evaluate the bilinearity level $\beta^{+}(\delta, \tau)$ for which the autoregressive forecasting statistic (7) can be used without significant increase in the mean-square risk.

\section{Numerical Results}

Monte Carlo numerical simulations were organized to illustrate the obtained theoretical formulas. The concrete model was considered:

$$
\begin{aligned}
x_{t}= & -0.5 x_{t-1}-0.5 x_{t-2}+0.1 x_{t-3}+0.2 x_{t-4}-0.2 x_{t-5}-0.1 x_{t-6} \\
& -0.2 x_{t-7}+0.2 x_{t-8}+0.1 x_{t-9}-0.1 x_{t-10}+\beta x_{t-1} u_{t-1}+u_{t},
\end{aligned}
$$

where $\left\{u_{t}\right\}_{t \in Z}$ are independent identically distributed as $N(0,1)$.

The scalar risks of the autoregressive forecasting statistic for the bilinear model were estimated, as well as the limits of $95 \%$ confidence intervals. The dependence on the bilinearity level and on the forecast horizon are shown on Figures 1 and 2 respectively. Figure 


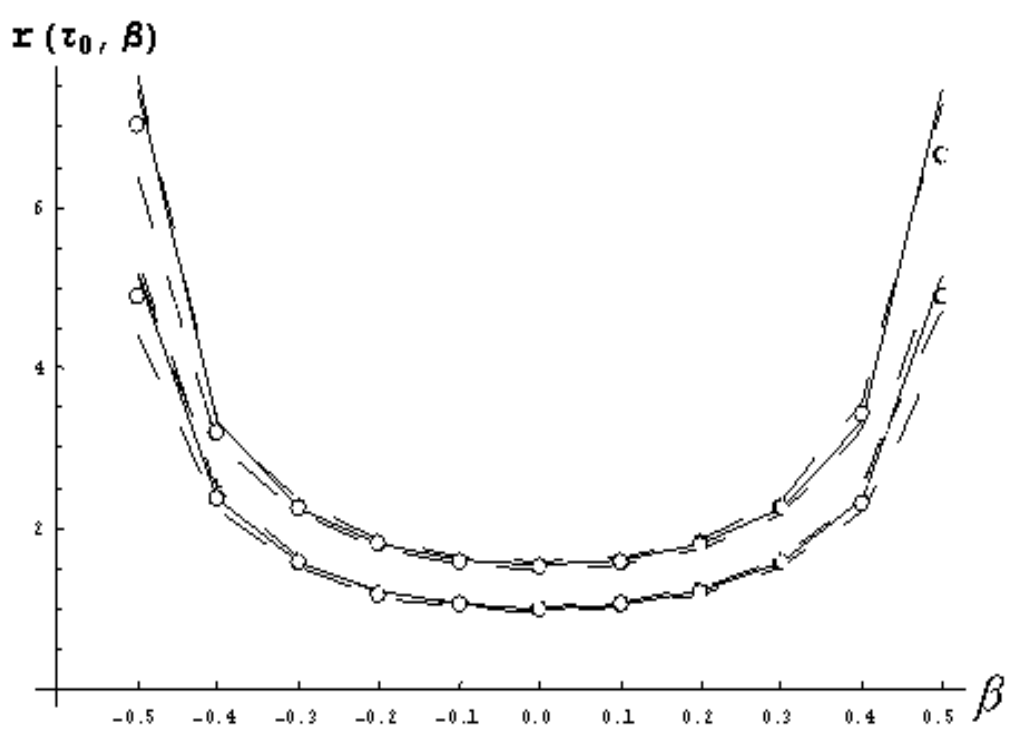

Figure 1: The exact risk and its point and 95\%-interval estimates at $\tau=1.5$

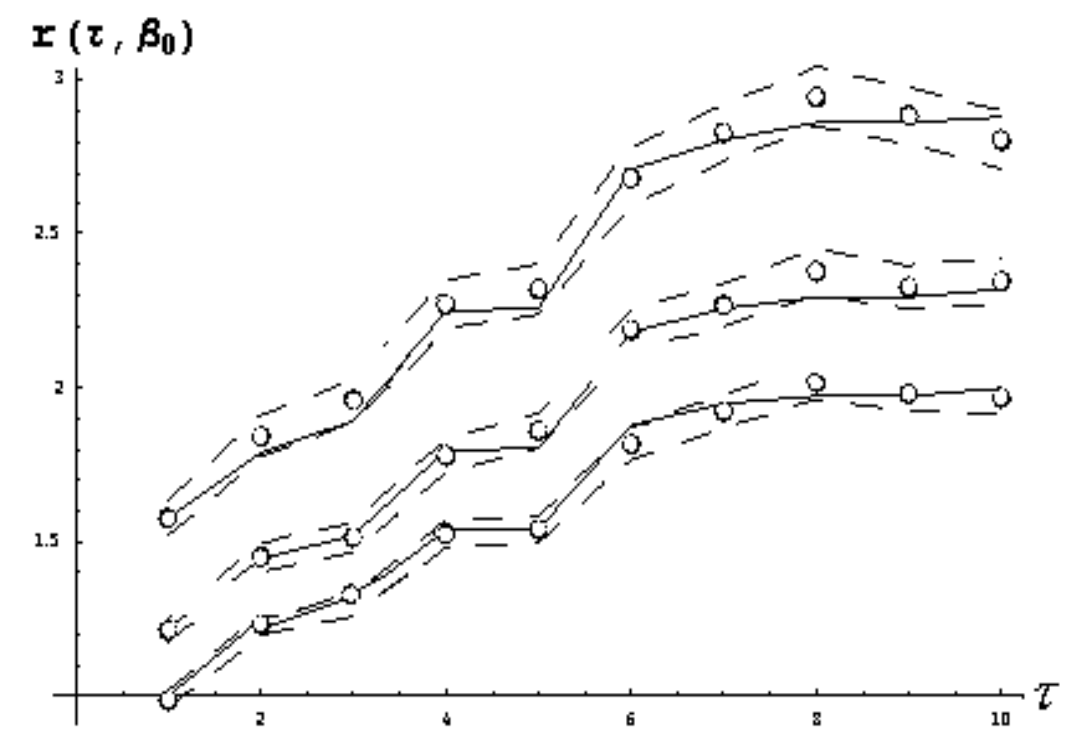

Figure 2: The exact risk and its point and 95\%-interval estimates at $\beta=0,0.2,0.3$

3 illustrates the accuracy of asymptotic approximation. Here the solid lines represent the exact or asymptotic risks computed by the formula (9) or (11), the dashed lines are the limits of confidence intervals and the dots are the Monte Carlo estimates of risks.

It is obvious from the Figures 1,2 that the risk starts to increase very rapidly after $\beta$ comes over the $\delta$-critical level of distortions $\beta^{+}(\delta, \tau)$ computed by Theorem 4 (Table 1 ). Figure 3 shows that for small bilinearity level $\beta$ the main terms of asymptotic expansion provide us with quite accurate approximation of exact risks. 


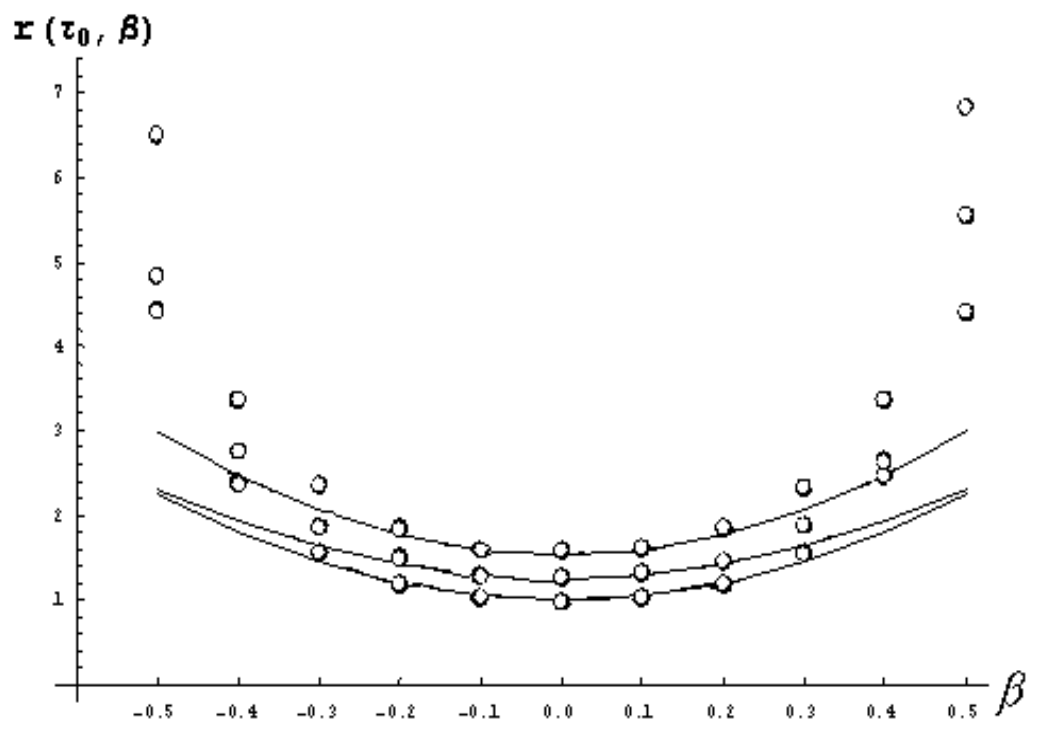

Figure 3: The asymptotic risk and the point estimates of exact risk at $\tau=1,2,5$

Table 1: The $\delta$-critical level of distortions

\begin{tabular}{cccc}
\hline$\tau$ & 1 & 2 & 5 \\
\hline$\delta=0.1$ & 0.141 & 0.171 & 0.163 \\
$\delta=0.5$ & 0.316 & 0.383 & 0.364 \\
$\delta=1.0$ & 0.447 & 0.542 & 0.515 \\
\hline
\end{tabular}

\section{Conclusion}

The paper contributes to the sensitivity analysis of the mean-square risk of the so-called "autoregressive forecasting statistic" (constructed for the hypothetical model AR(p)) in the situation where the observed time series satisfies really the model $\mathrm{BL}(\mathrm{p}, 0,1,1)$ with a small bilinearity coefficient $\beta$. Exact values and asymptotic expansions (at $\beta \rightarrow 0$ ) of matrix and scalar risks allow to find quantitative estimates of robustness and supply a statistician with the $\delta$-critical (for the given $\delta>0$ ) bilinearity level $\beta^{+}(\delta, \tau)$ satisfying the $\delta \cdot 100 \%$-admissible increment of the risk. Numerical results are in a good coincidence with the approximations of the risk generated by main terms of asymptotic expansions.

\section{References}

Anderson, T. W. (1994). The Statistical Analysis of Time Series. New York: WileyInterscience.

Fan, J., and Yao, Q. (2003). Nonlinear Time Series. Nonparametric and Parametric Methods. New York: Springer-Verlag. 
Granger, C., and Andersen, A. (1978). An Introduction to Bilinear Time Series Models. Gottingen: Vandenhoek and Ruprecht.

Kharin, Y. (1996). Robustness in Statistical Pattern Recognition. Dordrecht, Boston, London: Kluwer Academic Publishers.

Rao, S., and Gabr, M. (1984). An Introduction to Bispectral Analysis and Bilinear Time Series Models. New York: Springer-Verlag.

Terdik, G. (1999). Bilinear Stochastic Models and Related Problems of Nonlinear Time Series Analysis: A Frequency Domain Approach. New York: Springer-Verlag.

Authors' Address:

Yuriy Kharin and Olga Radzieuskaya

Department of Mathematical Modeling and Data Analysis

Faculty of Applied Mathematics and Informatics

Belarusian State University

Independence avenue, 4

Minsk 220030

Belarus

E-mail: kharin@bsu.by and radzieuskaya@gmail.com 